\title{
Intelligent algorithm to predict the spray deposition of UAV
}

\author{
Zhihong Huang ${ }^{1,2}$, Bo Zhang ${ }^{1,2^{*}}$, Yubin Lan ${ }^{2}$, Lili Wu ${ }^{1,2}$ \\ (1. Modern Education and Technology Center, South China Agricultural University, Guangzhou 510642, China; \\ 2. National Center for International Collaboration Research on Precision Agricultural Aviation Pesticides Spraying Technology (NPAAC), \\ Guangzhou 510642, China)
}

\begin{abstract}
Scientific use of pesticides can not only improve the effective utilization rate, but also reduce environmental pollution. As an important index to evaluate the effective utilization of pesticides, it is very important to measure the deposition of pesticides on crop surface quickly and accurately. Compared with the traditional manual sampling or sensor detection methods, the linear relationship model between the operating parameters such as flight speed, total flow rate of nozzle, spray volume per mu, spray amplitude and pesticide deposition by collecting spray parameters of UAV for plant protection was proposed, and the spray deposition of different parts of the wheat was predicted. Comparing with the collected samples data, the predicted results of the model shows that the intelligent algorithm is feasible and effective. It can provide an important basis for reducing pesticide application and further achieving precision spraying.
\end{abstract}

Keywords: intelligent algorithm, plant protection UAV, spraying, deposition, real-time prediction

DOI: $10.33440 /$ j.jpaa.20190202.37.

Citation: Huang Z H, Zhang B, Lan Y B, Wu L L. Intelligent algorithm to predict the spray deposition of UAV. Int J Precis Agric Aviat, 2019; 2(2): 49-53.

\section{Introduction}

Although China is a large agricultural country, its per capita cultivated land area is lower than the world average level due to its population ranking first in the world ${ }^{[1,2]}$. In order to prevent the damage of diseases and insect pests on crops and ensure the yield of crops, pesticides and chemical fertilizers have been widely used in agricultural production. Excessive use of pesticides and fertilizers not only destroys the ecological balance, but also is not conducive to the sustainable development of agriculture ${ }^{[3-6]}$. In order to effectively solve this problem, the government issued the plan for zero growth of pesticide use by 2020 on February 17, 2015, aiming to improve the effective utilization rate of pesticides, reduce agricultural production costs, protect the natural environment and promote the sustainable development of ecological environment ${ }^{[7]}$.

In the process of agricultural production, the effect of pesticide deposition, as an important index to evaluate the efficiency of pesticide use, has become an important index value that must be collected to promote green agricultural production ${ }^{[8-13]}$. Traditional detection methods of pesticide deposition amount include elution method and water sensitive paper method. This method mainly measures the deposition amount after spraying operation. Although the detection accuracy of this method is high, it cannot provide the deposition amount data in real time during the operation process, resulting in uneven spraying and repeated use in some regions, resulting in environmental pollution ${ }^{[14-17]}$. With the rapid development of Internet of things technology, sensor technology has been gradually introduced into the detection of

Received date: $2019-11-25$ Accepted date: 2019-12-15

Biographies: Zhihong Huang, Master, Senior engineer, research interests: agricultural aerial big data applications, Email: huangzh@scau.edu.cn; Yubin Lan, PhD, Distinguished Professor, Director, research interests: precision agricultural aviation application, Email: ylan@scau.edu.cn; Lili Wu, Master, Experimenter, research interests: agricultural aerial big data applications, Email: wll@scau.edu.cn.

* Corresponding author: Bo Zhang, PhD, Senior engineer, research interests: agricultural aerial big data applications, Email: zb@scau.edu.cn. pesticide deposition. This method realizes the real-time detection of deposition in the process of operation and provides an important foundation for precise spraying. However, due to the research of real-time sensor detection technology for the deposition of UAV spraying is still in its infancy, and affected by its own accuracy and other issues, it has not been widely used Extensive use requires continuous improvement ${ }^{[18,19]}$.

Based on the artificial intelligence algorithm, the linear relationship between the operational parameters of UAV, such as flight speed, total flow of nozzles, number of nozzles, spray volume per mu and spray amplitude, and the pesticide deposition amount of wheat ear, upper part, middle part and lower part was established by analyzing the collected data of UAV plant protection operation Measurement. This method can help to achieve the precise control of the dosage before the UAV plant protection operation, avoid repetitive operation, reduce the operation cost, reduce environmental pollution, make up for the shortcomings of laboratory and sensor detection methods, and improve the operation efficiency.

\section{Materials and methods}

\subsection{Experimental data}

The data of this study come from the operation data of spraying Bainongaikang 58 wheat in the period of flowering and filling by using plant protection UAV in Xinxiang comprehensive experimental base of Chinese Academy of Agricultural Sciences on April 20-21, 2017. The data of drug deposition was measured after the operation. The operation data include UAV flight speed, total flow rate, number of nozzles, spray volume per mu and spray amplitude. The amount of liquid medicine deposition in each part was obtained by the method of tracer measurement. This method adds a certain concentration of alluring red solution to the liquid medicine, and then spray it. Through elution, the absorption value of alluring red on crops is measured by spectrophotometer, according to the standard Curve calculation of deposition. In this study, 60 groups of liquid medicine deposition in different parts of wheat were selected as experimental data, including 15 groups in 
ear, upper, middle and lower parts.

\subsection{Prediction model of liquid medicine deposition}

Due to the small sample size, it is suitable to select machine learning mode for analysis. The multiple linear regression algorithm [20,21] is selected, and the UAV flight speed, total nozzle flow, number of nozzles, Mu spray volume and spray amplitude are used as the independent variable input models to respectively predict the liquid deposition of wheat ear, upper part, middle part and lower part. In the model, $X$ and $Y$ represent input and output variables respectively. In multiple linear regression, they are multidimensional vectors, as shown below:

$$
X=\left[\begin{array}{lll}
x_{1}^{1} & x_{1}^{2} & x_{1}^{n} \\
x_{2}^{1} & x_{2}^{2} & x_{2}^{n} \\
x_{m}^{1} & x_{m}^{2} & x_{m}^{n}
\end{array}\right]
$$

where, $x^{1}$ represents the flight speed; $x^{2}$ represents the total flow of nozzles; $x^{3}$ represents the number of nozzles; $x^{4}$ represents the spray volume per mu, and $x^{5}$ represents the spray amplitude.

$$
\theta=\left[\begin{array}{c}
\theta_{1} \\
\theta_{2} \\
\theta_{m}
\end{array}\right]
$$

where, $\theta$ represents the coefficient of the fitting function.

$$
Y=\left[\begin{array}{l}
y_{1} \\
y_{2} \\
y_{m}
\end{array}\right]
$$

where, $y$ is the deposition.

$$
Y=X \cdot \theta
$$

Formula (4) is a fitting function of the algorithm, which can be decomposed into polynomials as follows:

$$
\hat{y}^{i}(x)=\theta_{0} x_{0}+\theta_{1} x_{1}+\theta_{2} x_{2}+\ldots+\theta_{n} x_{n}
$$

where, $\hat{y}^{i}$ is the predicted value; $x_{0}$ is the constant 1 , and $x_{n}$ is the input independent variable.

For a single piece of data, the relationship between the real value and the predicted value is as follows:

$$
j(i)=y^{i}-\hat{y}^{i}
$$

where, $j(i)$ represents the difference between the real value and the predicted value, and the smaller the value is, the better. For the total data set, when the square function of the error, namely the loss function, reaches the minimum value, the model converges, and the loss function formula is as follows:

$$
J=\frac{1}{2 m} \sum_{i=0}^{m}\left(y^{i}-\hat{y}^{i}\right)^{2}
$$

where, $J$ represents the loss function value. The goal is to find a set of suitable fitting function coefficients $\theta$, so that $J$ value is as small as possible, that is, the predicted result after fitting is closest to the real value.

\section{Prediction and analysis}

\subsection{Sedimentation prediction}

The 60 groups of data selected are divided into four parts, i.e. wheat ear, upper part, middle part and lower part, according to the different collection positions of chemical deposition. Each part has 15 groups of data, 10 of which are training data sets and 5 of which are test data sets. The agent deposition prediction algorithm is divided into three stages: initialization data, training model and prediction deposition. The specific flow is shown in Figure 1.

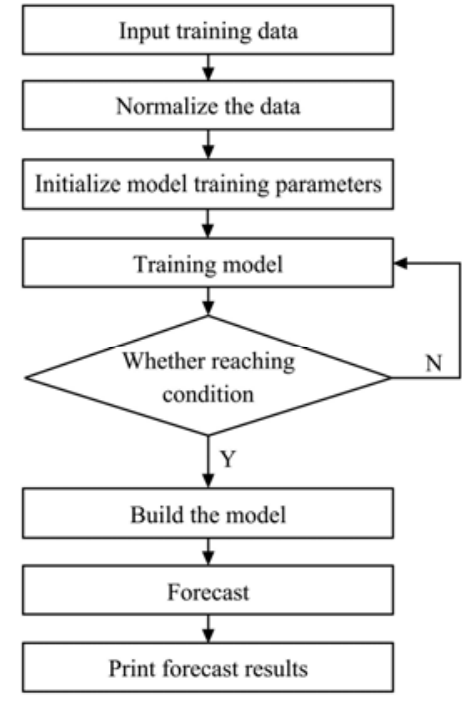

Figure 1 Algorithm flow chart

A. Phase 1

Phase 1 mainly completes data initialization, including normalization of training data set and initialization of fitting function coefficient.

Table 1 Data initialization of the first stage of multiple linear regression algorithm

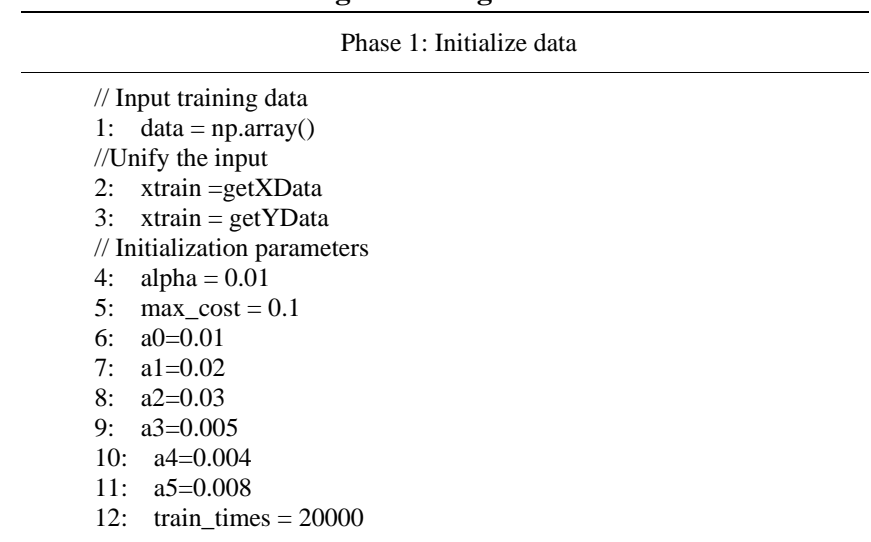

Line 1 completes the input of training data set, and lines 2-3 completes the normalization of training data set. The formula is as follows:

$$
\widehat{v}_{l}=\frac{v_{i}}{\max (v)}
$$

where, $\widehat{v}_{l}$ is the value after normalization; $v_{i}$ is the original value, and $\max (v)$ is the maximum value of the data.

Lines 4-11 initialize the fitting function coefficient $\theta$ to a random number close to 0 . Line 12 sets the number of model training to 20000 .

B. Phase 2

Phase 2 optimizes the value of fitting function coefficient $\theta$ through continuous iteration. In this study, when the iteration error is 0.1 , or the iteration reaches 20000 times, $J\left(\theta_{1} \theta_{2} \theta_{n}\right)$ obtains the expected value, achieves convergence and completes the model construction.

The updating process of the fitting function coefficient $\theta$ is as follows:

1) Substitute $\theta$ into formula (5) to calculate the predicted value;

2) Substitute the predicted value and the real value into formula (6) to get the error;

3) Put the error into formula (7) to calculate the total loss value;

4) The loss function $J\left(\theta_{1} \theta_{2} \theta_{n}\right)$ is derived. The specific 
derivation process is as follows:

$$
\begin{aligned}
& \frac{\partial}{\partial \theta_{j}} J(\theta)=\frac{\partial}{\partial \theta_{j}} \frac{1}{2 m} \sum_{i=0}^{m}\left(y_{i}-\hat{y}^{i}\right)^{2} \\
& =\frac{1}{m}(\hat{y}(x)-y) \cdot \frac{\partial}{\partial \theta_{j}}(\hat{y}(x)-y) \\
& =\frac{1}{m}(\hat{y}(x)-y) \cdot \frac{\partial}{\partial \theta_{j}}\left(\sum_{i=0}^{m} \theta_{i} x_{i}-y\right) \\
& =\frac{1}{m}(\hat{y}(x)-y) \cdot \frac{\partial}{\partial \theta_{j}}\left(\sum_{i=0}^{m} \theta_{i} x_{i}-y\right) \\
& =\frac{1}{m}(\hat{y}(x)-y) \cdot x_{j}
\end{aligned}
$$

5) Through derivation, the renewal formula of gradient decreasing $\theta$ is obtained. The specific formula is as follows:

$$
\hat{\theta}_{j}=\theta_{j}+\propto \cdot \frac{1}{m} \sum_{i=0}^{m}\left(y^{i}-\hat{y}^{i}\right) \cdot x_{j}^{i}
$$

where, $\propto$ is the learning rate. In order to achieve better optimization effect, $\propto$ is taken as 0.005 in this study.

Table 2 Phase 2 model construction of multiple linear regression algorithm

\begin{tabular}{l} 
Phase 2 : model building \\
\hline //Cycle training model \\
1: $\quad$ for $\mathrm{i}$ in range (0, train_times): \\
//Coefficient optimization of fitting function \\
2: $\quad$ optimize() \\
3: $\quad$ cost $=$ cost() \\
4: $\quad$ if(cost $<=$ max_cost): \\
$5: \quad$ break
\end{tabular}

\section{Phase 3}

Phase 3 is to predict the deposition of medicament. By setting the input data and substituting the data into the trained model, the prediction results are obtained.

Table 3 Prediction of the third stage chemical deposition by multiple linear regression algorithm

Phase 3: agent deposition prediction

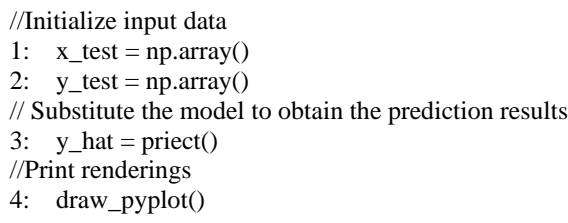

\subsection{Results and analysis}

Table 4-7 and Figure 2-5 show the real value, predicted value, predicted error, error rate and predicted effect chart of the five groups of test data sets of wheat ear, upper part, middle part and lower part, in which the ear error rate ranges from $4.35 \%$ to $12.83 \%$, the upper error rate ranges from $4.35 \%$ to $25.44 \%$, the middle error rate ranges from $0.78 \%$ to $15.69 \%$, and the lower error rate ranges from $0.78 \%$ to $15.69 \%$ in the range of $0.57 \%$ to $14.58 \%$, the overall effect of the prediction of the drug deposition in each part is middle $>$ lower $>$ ear $>$ upper. The prediction results of the chemical deposition amount of different parts of Wheat by multiple linear regression algorithm show that the prediction accuracy is

\begin{tabular}{|c|c|c|c|c|c|c|c|}
\hline \multirow{2}{*}{ Serial number } & \multirow{2}{*}{$\begin{array}{l}\text { True value } \\
/ \mu \mathrm{g} \cdot \mathrm{ear}^{-1}\end{array}$} & \multicolumn{3}{|c|}{ Multiple linear regression } & \multicolumn{3}{|c|}{ Neural network } \\
\hline & & Predicted value $/ \mu \mathrm{g} \cdot$ ear $^{-1}$ & Error value & Error rate & Predicted value $/ \mu \mathrm{g} \cdot \mathrm{ear}^{-1}$ & Error value & Error rate \\
\hline 1 & 44.49 & 41.76152309 & -2.72847691 & $-6.13 \%$ & 52.985363 & 8.495363 & $19.09 \%$ \\
\hline 2 & 83.49 & 73.63523411 & -9.85476589 & $-11.80 \%$ & 66.75068 & -16.73932 & $-20.05 \%$ \\
\hline 3 & 50.02 & 52.19349221 & 2.17349221 & $4.35 \%$ & 72.94962 & 22.92962 & $45.84 \%$ \\
\hline 4 & 66.85 & 75.42877767 & 8.57877767 & $12.83 \%$ & 81.912155 & 15.062155 & $22.53 \%$ \\
\hline 5 & 94.56 & 103.3897744 & 8.82977442 & $9.34 \%$ & 88.66173 & -5.89827 & $-6.24 \%$ \\
\hline
\end{tabular}
high and the prediction effect is good. The model is suitable for the small sample prediction of the chemical deposition amount of

\begin{tabular}{|c|c|c|c|c|c|c|c|}
\hline \multirow{2}{*}{ Serial number } & \multirow{2}{*}{$\begin{array}{l}\text { True value } \\
/ \mu \mathrm{g} \cdot \mathrm{cm}^{-2}\end{array}$} & \multicolumn{3}{|c|}{ Multiple linear regression } & \multicolumn{3}{|c|}{ Neural network } \\
\hline & & Predicted value $/ \mu \mathrm{g} \cdot \mathrm{cm}^{-2}$ & Error value & Error rate & Predicted value $/ \mu \mathrm{g} \cdot \mathrm{cm}^{-2}$ & Error value & Error rate \\
\hline 1 & 1.08 & 1.12700582 & 0.04700582 & $4.35 \%$ & 0.57966423 & -0.50033577 & $-46.33 \%$ \\
\hline 2 & 1.69 & 1.60573885 & -0.08426115 & $-4.99 \%$ & 1.2779742 & -0.4120258 & $-24.38 \%$ \\
\hline 3 & 2.57 & 1.91616681 & -0.65383319 & $-25.44 \%$ & 2.0314667 & -0.5385333 & $-20.95 \%$ \\
\hline 4 & 1.2 & 1.35928207 & 0.15928207 & $13.27 \%$ & 0.88347805 & -0.31652195 & $-26.38 \%$ \\
\hline 5 & 1.96 & 1.86774376 & -0.09225624 & $-4.71 \%$ & 1.6713597 & -0.2886403 & $-14.73 \%$ \\
\hline
\end{tabular}
wheat UAV after the application of chemical in the flowering and filling stage.

\begin{tabular}{|c|c|c|c|c|c|c|c|}
\hline \multirow{2}{*}{ Serial number } & \multirow{2}{*}{$\begin{array}{l}\text { True value } \\
/ \mu \mathrm{g} \cdot \mathrm{cm}^{-2}\end{array}$} & \multicolumn{3}{|c|}{ Multiple linear regression } & \multicolumn{3}{|c|}{ Neural network } \\
\hline & & Predicted value $/ \mu \mathrm{g} \cdot \mathrm{cm}^{-2}$ & Error value & Error rate & Predicted value $/ \mu \mathrm{g} \cdot \mathrm{cm}^{-2}$ & Error value & Error rate \\
\hline 1 & 0.41 & 0.38906452 & -0.02093548 & $-5.11 \%$ & 0.35841328 & -0.05158672 & $-12.58 \%$ \\
\hline 2 & 0.37 & 0.34443787 & -0.02556213 & $-6.91 \%$ & 0.22546603 & -0.14453397 & $-39.06 \%$ \\
\hline 3 & 0.35 & 0.40491978 & 0.05491978 & $15.69 \%$ & 0.34770623 & -0.00229377 & $-0.66 \%$ \\
\hline 4 & 0.42 & 0.45266643 & 0.03266643 & $7.78 \%$ & 0.45836473 & 0.03836473 & $9.13 \%$ \\
\hline 5 & 0.48 & 0.48376021 & 0.00376021 & $0.78 \%$ & 0.7075202 & 0.2275202 & $47.40 \%$ \\
\hline
\end{tabular}

Table 4 Prediction results of ear part

Table 5 Prediction results of upper part

Table 6 Prediction results of middle part 
Table 7 Prediction results of lower part

\begin{tabular}{|c|c|c|c|c|c|c|c|}
\hline \multirow{2}{*}{ Serial number } & \multirow{2}{*}{$\begin{array}{l}\text { True value } \\
/ \mu \mathrm{g} \cdot \mathrm{cm}^{-2}\end{array}$} & \multicolumn{3}{|c|}{ Multiple linear regression } & \multicolumn{3}{|c|}{ Neural network } \\
\hline & & Predicted value $/ \mu \mathrm{g} \cdot \mathrm{cm}^{-2}$ & Error value & Error rate & Predicted value $/ \mu \mathrm{g} \cdot \mathrm{cm}^{-2}$ & Error value & Error rate \\
\hline 1 & 0.31 & 0.31177303 & 0.00177303 & $0.57 \%$ & 0.2821555 & -0.0278445 & $-8.98 \%$ \\
\hline 2 & 0.24 & 0.27499332 & 0.03499332 & $14.58 \%$ & 0.1813131 & -0.0586869 & $-24.45 \%$ \\
\hline 3 & 0.35 & 0.32405745 & -0.02594255 & $-7.41 \%$ & 0.29374218 & -0.05625782 & $-16.07 \%$ \\
\hline 4 & 0.39 & 0.3634546 & -0.0265454 & $-6.81 \%$ & 0.35331059 & -0.03668941 & $-9.41 \%$ \\
\hline 5 & 0.36 & 0.3888288 & 0.0288288 & $8.01 \%$ & 0.5181992 & 0.1581992 & $43.94 \%$ \\
\hline
\end{tabular}

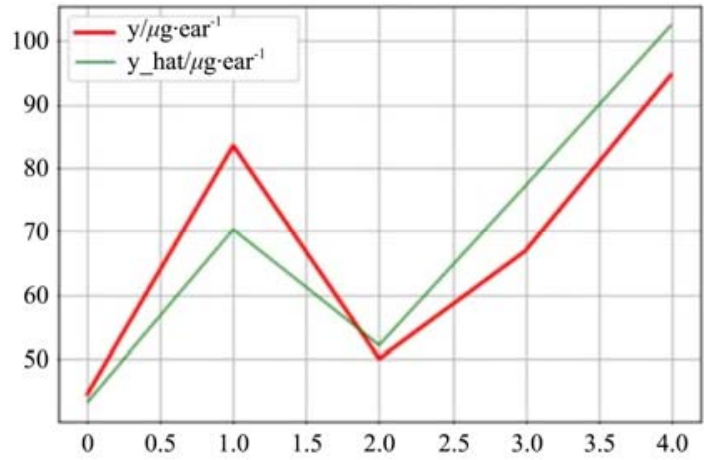

Figure 2 Effect diagram of ear part prediction

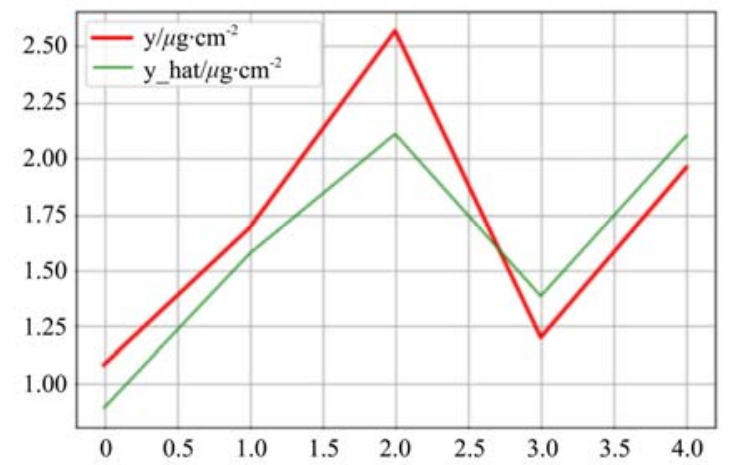

Figure 3 Effect diagram of upper part prediction

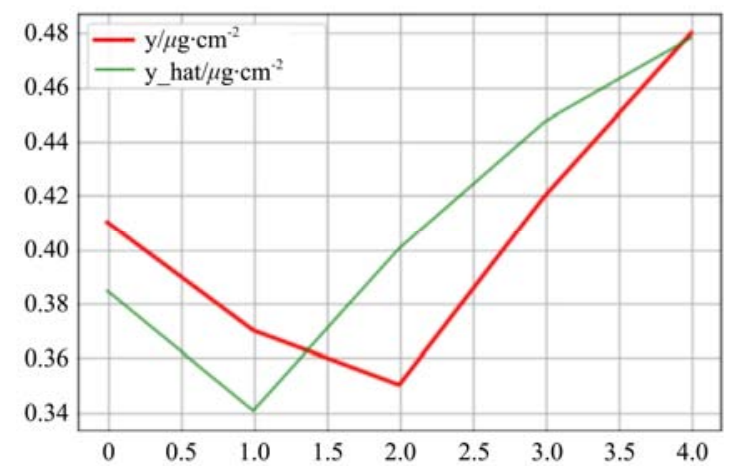

Figure 4 Effect diagram of middle part prediction

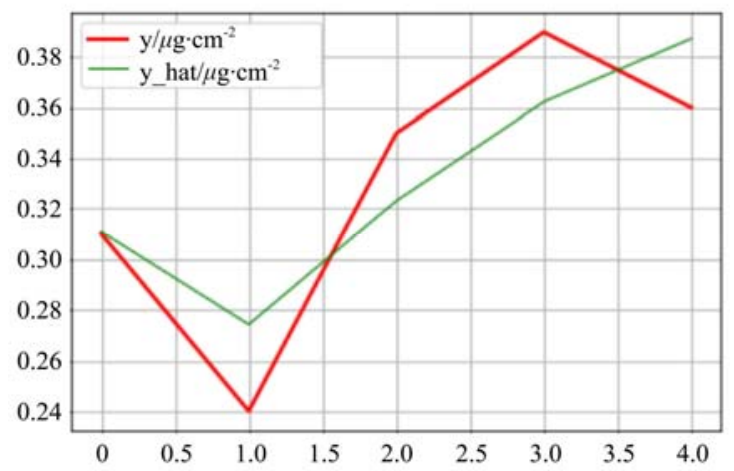

Figure 5 Effect diagram of lower prediction
The study selects the neural network algorithm and multiple linear regression algorithm to conduct comparative experiments. According to Tables 4-7, under the same experimental environment and test data scale, the error of the deposition amount of each part of wheat predicted by the neural network algorithm is greater than the multiple linear regression algorithm used in this study.

\section{Conclusions}

We have proposed a prediction model of the deposition amount of the spray liquid of UAV, based on multiple linear regression algorithm, the speed, the total flow rate, the number of sprayers, the amount of spray per mu, the spray amplitude and the amount of liquid deposition in different parts of the 60 groups of wheat were trained by using the operation data of the UAV to spray the wheat during the flowering and filling period and the amount of liquid deposition in each part of the 60 groups of wheat. Therefore, we can use the spraying data of UAV for plant protection to predict the deposition of chemical solution after operation, and provide important basis for pesticide reduction and precision spraying.

However, the research data is mainly from an experiment, so the influence of the number of samples, different growth periods of crops, different meteorological environment and other factors has not been considered in the algorithm. The future work will focus on solving these problems. Through the continuous improvement of the influence factors and the continuous optimization of the algorithm, a more accurate prediction model for the deposition amount of the spray solution of UAV will be built on the basis of multiple samples.

\section{Acknowledgements}

We deeply thank for the National Key Technologies Research and Development Program (2016YFD0200801), Guangdong Leading Talent Project (2016LJ06G689), National Key Technologies Research and Development Program (2016YFD0200700). Thanks to all the participants who provided the experimental data for this study (Lan Yubin, Meng Yanhua, Li Jiyu, Song Jianli, Yan Xiaojing, Ma Yan, Cai Chen, Wu Qiufang, Zhou Qingqing, Zhang Lei, Ren Xiangliang, Hu Hongyan, Wang Guobin, Huang Huasheng, Wang Shilin, Wang Zhiyi, Chen Pengchao, Ali, Li Yifan, Suo Gaoyu, Deng Yusen, Zhou Yangyang, Kong Xiao, Zhou Xiaoxin, Tang Xiujun, Wang Xueliang, Wang Jing, Shi Haotian, Zhang Zhixian, Fan Weiyan, Hu Chao, Wang can, Li Yanshang, Cheng Yunwei, Fang Haijun, Wang $\mathrm{Pu}$ ). Thanks to national aviation plant protection science technology innovation alliance and Xinxiang comprehensive experimental base of Chinese Academy of Agricultural Sciences for strong support.

\section{[References]}

[1] Wang J, Hao J M, Chen A Q, et al. Supply and demand balance of ecological carrying capacity in provincial arable land of China. Journal of 
China Agricultural University, 2019; 24(07): 146-155. doi: 10.11841/ j.issn.1007-4333.2019.07.18.

[2] Ge D Z, Long H L, Yang R. The pattern and mechanism of farmland transition in China from the perspective of per capita farmland area. Resources Science, 2018; 40(02): 273-283. doi: 10.18402/resci.2018.02.05.

[3] Li Y J. Analysis on the double reduction of chemical fertilizer and pesticide. South China Agriculture, 2019; 13(05): 174-175. doi: 10.19415/j.cnki.1673-890x.2019.05.091.

[4] Dong M L. Study on pesticide pollution and environmental protection measures. Shanxi Agricultural Economy, 2018; (21): 117. doi: 10.16675/j.cnki.cn14-1065/f.2018.21.085.

[5] Sun W F, Wang L J, Chen B C, et al. The present situation and development of study on the pesticide applying technology at home and abroad. Journal of Agricultural Mechanization Research, 2009; 31(09): 225-228. doi: 10.3969/j.issn.1003-188X.2009.09.067.

[6] Shen Y. Residue characteristics and degradation mechanisms of common pesticides in wheat. Ph.D. dissertation, 2007; Yangzhou University. doi: 10.7666/d.y1103243.

[7] Liu G. Ministry of Agriculture issues action plan for zero growth of pesticide use by 2020. Pesticide Market News, 2015; (08): 10-12. doi: 10.13378/j.cnki.pmn.2015.08.003.

[8] Su X J, Wang Y L, Wei J, et al. Pesticide deposition percentage and control effect of nine kinds of crop protection machineries against wheat aphid. Acta Agriculturae Boreali-occidentalis Sinica, 2018; 27(01): 149-154. doi: 10.7606/j.issn.1004-1389.2018.01.020.

[9] Guan X B, Kong X, Qi P, et al. Effects of spraying mechanical spraying on pesticide deposition rate and control efficiency of wheat aphid. Agriculture of Henan, 2018; (18): 43-44, 46. doi: 10.15904/ j.cnki.hnny.2018.18.022.

[10] Yuan H Z, Yang D B, Yan X J, et al. Pesticide efficiency and the way to optimize the spray application. Plant Protection, 2011; 37(05): 14-20. doi: 10.3969/j.issn.0529-1542.2011.05.002.

[11] $\mathrm{Xu} \mathrm{D} \mathrm{J,} \mathrm{Xu} \mathrm{G} \mathrm{C,} \mathrm{Xu} \mathrm{X} \mathrm{L,} \mathrm{et} \mathrm{al.} \mathrm{Influence} \mathrm{of} \mathrm{spray} \mathrm{nozzle} \mathrm{and} \mathrm{spray}$ volume on pesticide deposition and control effect in rice. Journal of Plant Protection, 2019; 46(02): 409-416. doi: 10.13802/ j.cnki.zwbhxb.2019.2018006.
[12] Wang M. Determination and evaluation model of pesticide deposition rate for utilization by folia spray in rice field. Master dissertation, 2019; Chinese Academy of Agricultural Sciences.

[13] Hu H Y, Ren X L, Jiang W L, et al. Pesticide spray distribution of plant protection UVA in cotton field. Journal of Huazhong Agricultural University, 2018; 37(05): 59-64. doi: 10.3969/ j.issn.1000-1190.2018.05.009.

[14] Liu Y. Synergism mechanism of organic silicon and xanthan gum on fungicide. Master dissertation, 2010; Anhui Agricultural University. doi: 10.7666/d.y1735212

[15] Wang S, Peng E R, Wu G X, et al. Surveys of deposition and distribution pattern of pesticide droplets on crop leaves. Journal of Yunnan Agricultural University, 2010; 25(01): 113-117. doi: 10.3969/ j.issn.1004-390X.2010.01.020.

[16] Belden J B , Hanson B R , Mcmurry S T , et al. Assessment of the effects of farming and conservation programs on pesticide deposition in high plains wetlands. Environmental Science \& Technology, 2012, 46(6): 3424-3432. doi: 10.1021/es300316q.

[17] Zhang H Y, Lan Y B, Wen S, et al. Operational effects of unmanned helicopters for pesticide spraying in rice field. Journal of South China Agricultural University, 2019; 40(01): 116-124. doi: 10.7671/ j.issn.1001-411X.201802028.

[18] Zhang R R, Chen L P, Lan Y B, et al. Development of a deposit sensing system for aerial spraying application. Transactions of the Chinese Society for Agricultural Machinery, 2014; 45(08): 123-127. doi: 10.6041/ j.issn.1000-1298.2014.08.020.

[19] Wang F. Study on electrochemical sensing system for detection of pesticide spray deposition. Master dissertation, 2017; Jiangsu University.

[20] Fan L J. Energy consumption forecasting and energy saving analysis of urban buildings based on multiple linear regression model. Natural Science Journal of Xiangtan University, 2016; 38(01): 123-126. doi: 10.3969/j.issn.1000-5900.2016.01.026.

[21] Peng H, Zhao Y J, Hu Z H. Railway passenger volume forecast based on multiple linear regression model. Journal of Chongqing University of Technology (Natural Science), 2018; 32(09): 190-193. doi: 10.3969/ j.issn.1674-8425(z).2018.09.030. 